\title{
A Split Connection TCP Proxy in LTE Networks
}

\author{
Viktor Farkas, Balázs Héder, and Szabolcs Nováczki \\ Nokia Siemens Networks \\ \{viktor.farkas, balazs.heder, szabolcs.novaczki\}@nsn.com
}

\begin{abstract}
High quality and ubiquitous Internet access is a key feature of today's mobile systems such as LTE. While LTE can provide competitive peak data rates and a relatively low latency, there is still room for solutions improving end-users' Quality-of-Experience by optimizing services running over the LTE infrastructure. Being the most widespread transport protocol, TCP is in the main focus of such research projects. A widely recommended solution for TCP performance improvement is the split connection TCP proxy that divides the end-to-end TCP connection into two independent connections, that results increased throughput and faster error recovery. This paper investigates the performance of a split connection TCP proxy deployed in LTE's SAE-GW. Numerical results show significant performance improvement of file downloading, web browsing and video steaming applications in case of not congested transport networks.
\end{abstract}

Keywords: LTE, PEP, TCP proxy, split TCP.

\section{Introduction}

Latest advances in cellular mobile technology enables users to access most of their favorite services and applications with competitive quality compared to fixed access alternatives. Long Term Evolution (LTE) is the latest 3GPP standard, offering high peek data rates and low latency, currently under deployment by many cellular network operators. The enhanced system capacity enables LTE systems to be a valid alternative of fixed Internet access with the added value of mobility. However, high data rate and low latency provided by LTE infrastructure are rarely enough to gain end-user satisfaction without higher layer services being able to adapt to the environment and utilize resources in an optimal way.

Transmission Control Protocol (TCP) [6] is used by various Internet services that require reliable end-to-end data transport for content delivery. Since TCP is the dominant transport protocol of Internet based applications, its performance plays a critical role in the user experience. Reliable and efficient data transfer requires that TCP is able to dynamically adapt its rate to the available resources and that it is capable to detect data loss in order to retransmit the missing data. The rate adaptation is handled by TCP's congestion control (CC) algorithm. The main assumption of the TCP CC algorithm - that data loss is due to congestion and not to bit errors - is valid in case of wired systems; however, limitations 
introduced by the radio interface (large round-trip time (RTT), fluctuation of the RTT, and transmission errors due to the imperfections of the wireless link) make TCP non-optimal for transferring data over mobile networks [28].

The performance improvement of the TCP protocol over wireless links has been a subject of intensive research in recent years. Proposals can be grouped into two categories: end-to-end and network supported solutions. End-to-end solutions require changes in the client and server TCP implementations, whereas network supported solutions require extra functionality at the underlying network. Important examples of end-to-end solutions are TCP Eifel [16] and TCP Westwood [17]. The network supported solutions are commonly referred as Performance Enhancing Proxies (PEP) [5]. The most prominent implementation of the PEP concept is the split connection TCP proxy, which divides traversing TCP connections into two independent connections, while being transparent to the end-points. The TCP proxy can improve end-to-end performance of a TCP connection is two ways. On one hand a proxy can separate the transmission path along network boundaries with significantly different characteristics and quarantine transmission problems in the affected loop. A proxy deployed at the boundary of the wired and wireless part of a network can isolate the impacts of the air interface problems (i.e., TCP congestion control problems) from the rest of the system and handle these locally.

This paper evaluates the efficiency of the split connection TCP proxy concept in LTE systems with proxy deployed in the System Architecture Evolution Gateway (SAE-GW). The performance is evaluated in the context of three mobile applications, namely FTP based file transfer, web browsing and streaming video, in terms of end-to-end throughput, mean download time and user satisfaction, respectively. Numerical results are provided based on simulations.

The rest of the paper is organized as follows. In Section 2, an overview of the related work is presented, which is followed by an introduction of the split TCP proxy architecture in the LTE environment, along with the discussion of the possible deployment options in Section 3. The simulation model and the results of the simulations are discussed in Section 4. Finally, the paper is concluded in Section 5 .

\section{Related Work}

The concept and practical benefits of PEPs was in main focus of several research projects during the last decade. Many research papers investigate the split connection TCP proxy concept applied in case of satellite links that have high RTT [8], ad-hoc networks with high bit error rate links [18], as well as in case of transport infrastructures for reliable overlay multicast networks 15. Lately several studies investigate the potential benefits of using the split TCP concept in wireless [27 1322] and in particular in cellular networks [14]. The authors in [19] give a comprehensive overview of split connection TCP proxies in Wideband Code Division Multiple Access networks and show that this solution can significantly improve the throughput of file downloads, while in [21] the performance 
(e.g., throughput, page download time) of Hypertext Transfer Protocol (HTTP) proxy and TCP proxy solutions is compared. In [7] a TCP proxy with flow aggregation capability is investigated applied in order to enhance the user experience in case of General Packet Radio Service data calls. A radio network feedback controlled TCP proxy is studied in [20] that has as scope to improve the end-to-end TCP performance in a $3 \mathrm{G}$ network. The radio quality feedback (e.g., radio bandwidth changes, queue length in the Radio Network Controller) is used to adapt the TCP window size in the proxy. A similar concept is studied in 25] in order to mitigate the effects of bandwidth oscillation in High-Speed Downlink Packet Access networks, while in [26] the same concept is empowered by an analytical model and further simulation results.

\section{TCP Proxy in LTE Networks}

\subsection{LTE Overview}

The LTE system is structured into four high level domains (as depicted in Figure 1): User Equipment (UE), Evolved UMTS Radio Access Network (E-UTRAN), Evolved Packet Core (EPC), and the Services domain [12]. The UE, E-UTRAN and EPC form the Internet Protocol (IP) Connectivity Layer, with the main function to provide IP based connectivity. E-UTRAN consists of the evolved Node Bs (eNBs). The EPC consists of the Mobility Management Entity (MME), which is the control plane functional element, and the SAE-GW (consisting of the Serving-GW and Packet-GW), which is the user plane gateway to the EUTRAN. The Services domain consists of subsystems that provide the various operator services or grants access to the external services such as those available on the Internet. The UEs are connected to the eNBs via the air (Uu) interface. eNBs are connected to the SAE-GW via the S1-U and to the MME via the S1-C interface. The MME and the SAE gateway communicate with each other via the S11 interface. User traffic is carried via the S1-U interface through General Packet Radio Service Tunneling Protocol (GTP) tunnels, whereas control plane traffic via the $\mathrm{S} 1-\mathrm{C}$ interface. The eNBs are interconnected via the $\mathrm{X} 2$ interface that is used in order to transfer the handover related user and control plane traffic.

\subsection{The Split Connection TCP Proxy Concept}

A split connection TCP proxy captures TCP session establishment attempts and creates two independent TCP connections working in tandem instead of one end-to-end session. The proxy transparently emulates the communication end-points so that the presence of the proxy is seamless to them. The two legs of the split connection are independent in means of flow and congestion control, while they are using a shared buffer to exchange client data.

The main property of a split TCP connection is that RTT is reduced in the split loops thus higher throughput can be achieved compared to the original 


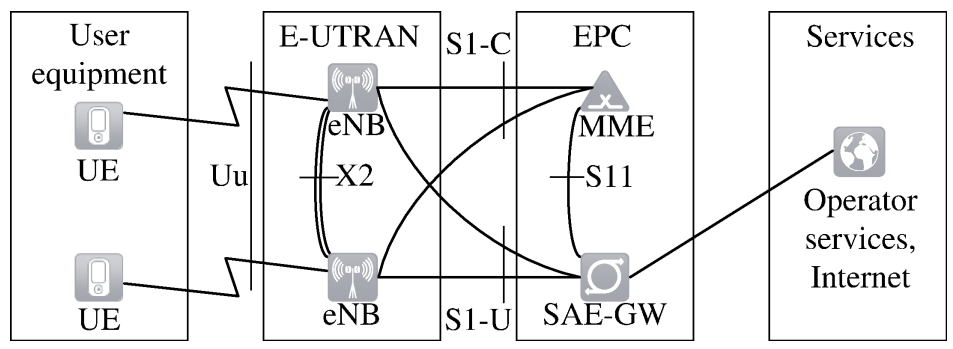

Fig. 1. The LTE network architecture

end-to-end throughput. As concluded in [3] the theoretical maximum end-to-end throughput is the maximum throughput of the loop with higher RTT (i.e., the one with smaller throughput). Reduced RTT enables higher maximum throughput in the split loops, which can be achieved faster as TCP can increase its congestion window size with an increased rate in all operation phases (slow start, congestion avoidance and fast recovery). Other main benefit of a split TCP connection is that effects of link failures on TCP performance are not propagated between the loops, while the affected loop can recover faster. The latter property can be highly beneficial in networks with wireless links where the main source of packet drop is not network congestion but poor radio quality. The latter can significantly deteriorate TCP performance as TCP assumes packet drops as indication of network congestion.

\subsection{Deployment Options}

The benefits highlighted above can be exploited by deploying a TCP proxy functionality in an LTE network, however the expected gains highly depend on its location. The possible locations of the proxy functionality are the SAE-GW (before S1 tunnel), the eNB (between the S1 tunnel and the Data Radio Bearer) and finally the combination of the above two. Each solution has its drawbacks and benefits, which are shortly outlined here.

The SAE-GW based solution (when the TCP proxy is located at the SAEGW) is the most straightforward option as the SAE-GW is the mobility anchor point for user plane traffic. The RTT ratio of the split loops is more auspicious as both split loops have smaller RTT than the original end-to-end RTT. As shown in Figure 2 the smaller RTT enables shorter TCP connection setup and data transfer times in slow-start mode. The drawback of this option is that air interface problems are recovered slower compared to the eNB based solution.

An eNB based TCP proxy is the deployment option if air interface problems need to be handled in a quick, low RTT radio loop and masked from the rest of the network. However, the implementation is not as straightforward as in case of the SAE-GW base solution as during handovers the TCP proxy context have to be transfered between the eNBs. Moreover, as the RTT in the other loop (i.e., towards the CS) is almost the same as the original end-to-end RTT, the eNB 
based TCP proxy cannot significantly increase end-to-end throughput in case of good radio conditions on the air interface.

A combined SAE-GW/eNB solution is expected to deliver best results, as it inherits the benefits of the base options while also complement each other. The eNB proxy provides fast recovery on the air interface while the SAE-GW boosts the end-to-end throughput. Note that the context transfers during handovers still has to be managed in the eNB proxy.

Due to its simple implementation (i.e., no handovers had to be considered) and its more general potential benefits (i.e., not bound to radio link failures) we have selected the SAE-GW based option for our first investigations, the results of which is presented in this paper. A SAE-GW side TCP proxy splits end-to-end TCP connections into two corresponding TCP loops: one between the CS and the SAE-GW and another between the SAE-GW and the UE. The TCP proxy emulates the UE's behavior towards the CS, while it faces as the CS towards the UE. The presence of the proxy is transparent both to the UE and the CS. Note, that the two TCP loops are independent from each other in means of flow and congestion control. Moreover they might use different TCP parameters or even variants. The loops are connected by a shared buffer in each direction. Data received from the CS is stored in the downlink shared buffer as long as it is forwarded to the UE, and a similar buffer is used for the uplink traffic. A backpressure mechanism [15] is used to prevent buffer overflows.

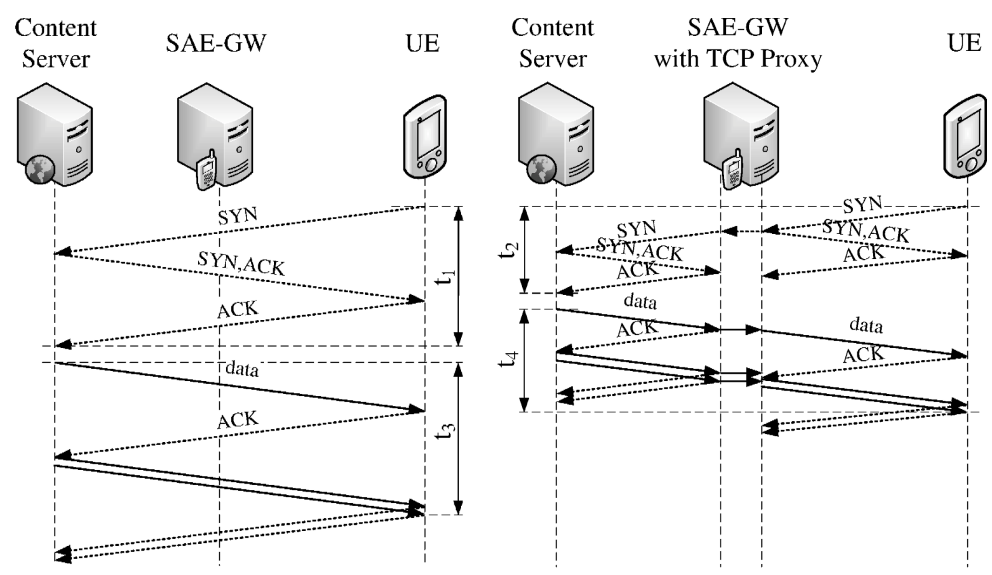

Fig. 2. Duration of TCP connection setup $\left(t_{1}\right.$ an $\left.t_{2}\right)$ and data transfer in slow start phase $\left(t_{3}\right.$ and $\left.t_{4}\right)$ with and without TCP proxy

\section{Simulation Results}

\subsection{The Simulation Environment}

The performance of the TCP proxy in LTE networks was investigated through simulations in an event based simulation environment. In the simulator a reference 
LTE network was created which simulates an urban environment with pedestrian users and average radio conditions. All simulations were executed in this reference LTE network. The radio network layout of the reference LTE network was designed according to the double ring network topology [1]; the 7 eNBs of the inner ring were serving the traffic, the outer ring of eNBs were added only for interference generation. In Figure 3 the topology of the reference LTE network is depicted. The 7 eNBs were connected to the SAE-GW through IP routers, which were connected via $100 \mathrm{Mbit} / \mathrm{s}$ Ethernet links with $10 \mathrm{~ms}$ media delay. The IP routers used the Random Early Detection (RED) queue management technique [10]. Each CS was connected through $100 \mathrm{Mbit} / \mathrm{s}$ Ethernet links with a fix delay to the SAE-GW, this delay is referred as the mean Internet delay in the rest of the paper. Each simulation case was executed with and without TCP proxy.

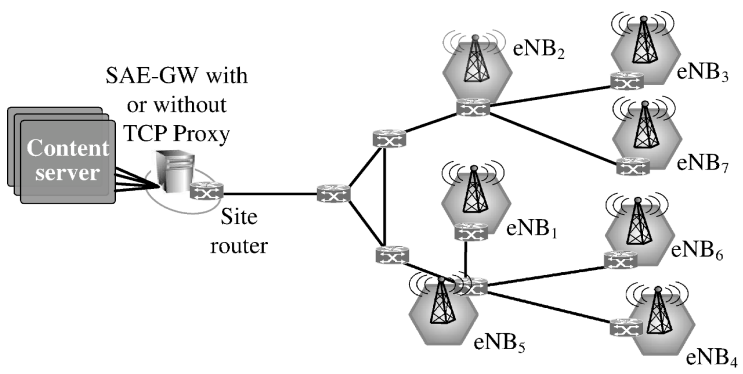

Fig. 3. The simulated topology

In the simulation environment the protocols of the S1-U and LTE-Uu interface are implemented in detail. The air interface is based on 3GPP 25.814 [1]. A single input single output channel with two times $10 \mathrm{MHz}$ bandwidth is considered. Each eNB is simulated with a one cell one sector configuration, having an omni-directional antenna. The mobility model is random way-point, inter-site handover procedures are implemented. The channel quality is simulated separately for each user considering distance loss, shadowing, multipath fading, and penetration loss.

Three key Internet applications were used as benchmark applications in order to evaluate the performance impacts of a TCP proxy in an LTE network: FTP based file transfer (bulk data transfer), HTTP traffic (web browsing) and video streaming. These three applications are implemented in detail in the simulation environment. Each user had one of these three applications running during the simulations. The TCP proxy was implemented in detail with two TCP variants: New Reno and CUBIC. TCP New Reno 9] is also referred as standard TCP because it is the most well-known TCP variant. TCP CUBIC [11] was developed for networks with high bandwith-delay product and it is the current default TCP variant of the Linux operating system so it is used by a large number of content servers and Android mobile devices. 
FTP users initiate a file download with unlimited file size. The measured performance indicator for FTP traffic is the average throughput and the fairness of the TCP connections as defined by the Jain's Fairness Index [23]. The Jain's Fairness Index of connections with equal throughput is 1; the higher is the difference in the throughput of different connections, the lower is the Jain's Fairness Index.

The simulation model of web browsing was based on HTTP/1.1. Pipelining, which has a large impact on the performance of mobile browsing was also part of the model [4. The browser client was modeled based on a typical mobile web browser. Web browsing users were acting according to the common web surfing behavior: they selected a random web page from a set of popular sites, downloaded them and proceeded to another random page after a simulated reading time. Web pages were selected from the top 20 sites of Alexa's top 500 global list [2]. The structure of these web sites (i.e., the number of embedded objects and the size of the HTTP request and response with the address of the server for each object) was recorded in September, 2011. This state was used in the simulations so that Domain Name System (DNS) queries, HTTP requests and HTTP responses could be simulated authentically. For HTTP users, the measured performance indicator is the mean page download time.

Video streaming was modeled with the web browsing model extended with the relevant features of the HTML5 (the fifth version of the HyperText Markup Language) standard. The downloaded video had a $512 \mathrm{kbit} / \mathrm{s}$ average video bitrate and it was $90 \mathrm{~s}$ long. Videos of this size are not transferred with server side rate control [24]. The performance measure in this case is the user satisfaction: during the simulations users were considered satisfied if the buffering time of the video was shorter than eight seconds and the video playback was not interrupted more than three times or for more than two seconds altogether.

\section{$4.2 \quad$ Numerical Results}

First, the performance of downlink bulk data transfer with and without a TCP proxy is evaluated. It is studied how the performance depends on the TCP variant, the mean Internet delay and the congestion level of the transport network. FTP simulations were executed with both the New Reno and the CUBIC TCP variant. For the mean Internet delay, three different values were set: 50, 75 and $125 \mathrm{~ms}$; these represent the cases of communicating with a server with low, medium and large delay. The different congestion levels of the transport network were simulated with different number of users per cell. The not congested case was modeled with 2 users per cell, the moderately congested case with 4 and 6 users per cell, and the congested case with 8 and 10 users per cell.

Figure 4 shows the per user average throughput and the Jain's Fairness Index in case of FTP data transfer with and without a TCP proxy when the mean Internet delay was set to $50 \mathrm{~ms}$. TCP proxy was able to increase the mean throughput by more than $60 \%$ where there was no congestion, by $2-17 \%$ in case of moderate congestion and by a few percents under heavy congestion. This is due to the fact that the TCP proxy is not able to increase the throughput 
of TCP connections if the transport network is congested, as in these cases the load is at the maximum. The TCP variant had minimal impact on the achievable throughput. The reason for this is that the bandwidth-delay product of the LTE system is not in the range where the TCP CUBIC is able to achieve a higher throughput than TCP New Reno. The Jain's Fairness Index was above 0.96 in all cases, which generally means a good fairness. However, the Fairness Index was a bit worse with TCP proxy than without TCP proxy. The reason for this is that during the simulations without TCP proxy a fix Internet delay is part of the RTT of end-to-end TCP loops. On the other hand during simulations with TCP proxy this fix delay is not part of the RTT of the SAE-GW-UE TCP loop. Thus the variance of the RTT is smaller in the case with TCP proxy than without TCP proxy.
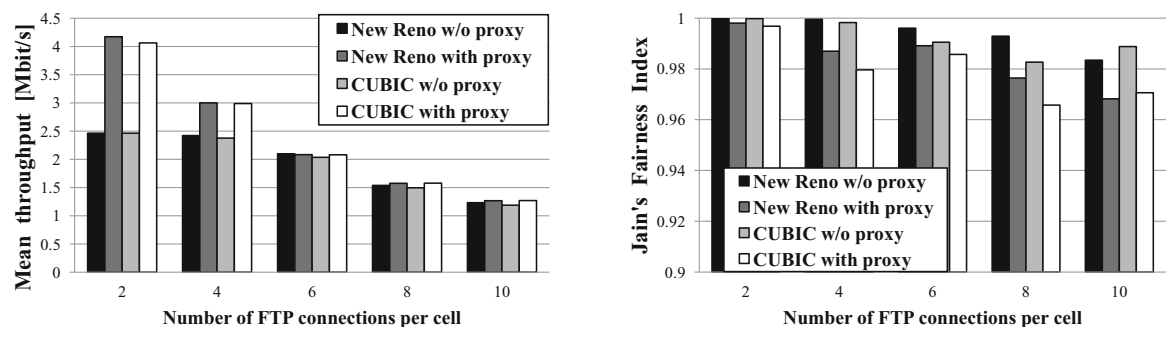

Fig. 4. Average throughput and Jain's Fairness Index for FTP data transfer with and without a TCP proxy when the mean Internet delay is $50 \mathrm{~ms}$
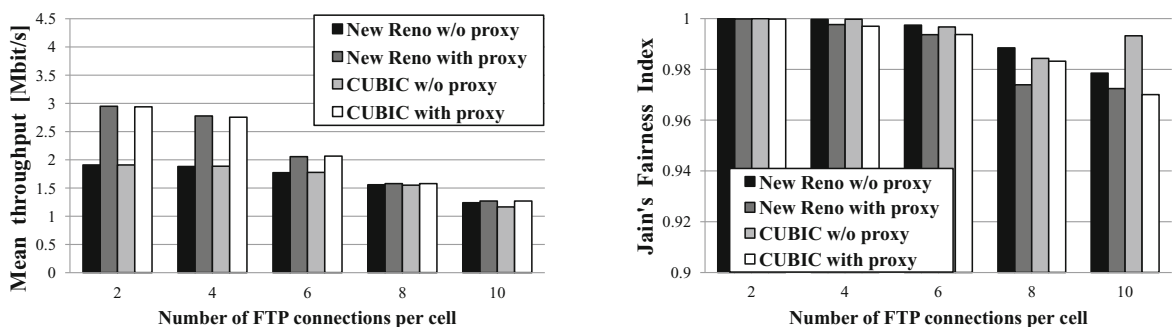

Fig. 5. Average throughput and Jain's Fairness Index for FTP data transfer with and without a TCP proxy when the mean Internet delay is $75 \mathrm{~ms}$

Figure 5] and 6] show the same diagrams for the cases where the mean Internet delay is 75 and $125 \mathrm{~ms}$. These figures indicate a moderate gain in throughput under light and moderate congestion. The smaller gain of TCP proxy in the not congested case is because the larger RTT between the CS and the SAEGW decreases the achievable maximum throughput on the corresponding TCP loop of TCP proxy. The larger increase in the moderately congested case is 

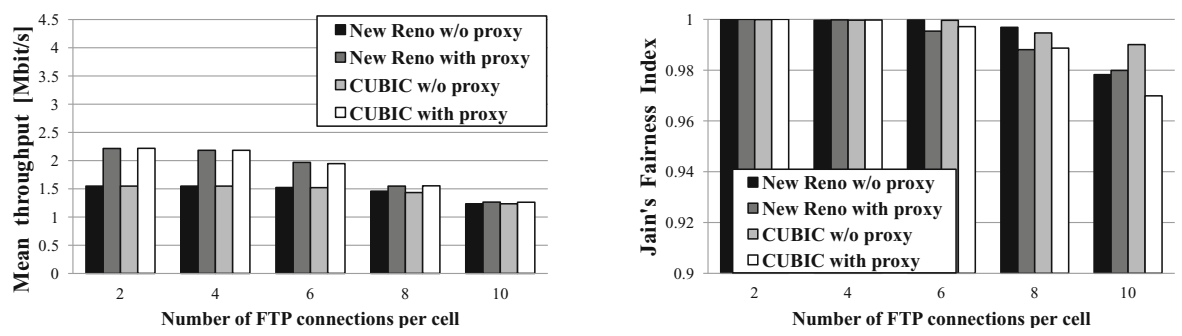

Fig. 6. Average throughput and Jain's Fairness Index for FTP data transfer with and without a TCP proxy when the mean Internet delay is $125 \mathrm{~ms}$

possible because the larger Internet delay degrades the achievable throughput of the reference cases, thus the transport network become fully utilized at a higher number of users per cell.

The performance of web browsing with and without a TCP proxy is presented for the New Reno TCP variant. All HTTP simulations were repeated with the CUBIC TCP variant as well but the performance difference between the two variants was minimal, so those results are not shown. The investigated mean Internet delay values were the same as for the FTP simulations. The congestion level of the network was changed by adding background FTP downloads; in the uncongested case, there were no background FTP downloads while 2 to 4 and 6 to 8 FTP downloads per cell simulated the moderately congested and congested cases. Every HTTP simulation case was executed with 5 different random seeds.

Figure 7 (a) shows the mean download times when the mean Internet delay was $50 \mathrm{~ms}$. It is clear that the TCP proxy decreases the average download time in all the investigated cases. As expected, the mean download time increases when the transport gets more congested but there is a benefit of a TCP proxy in those cases as well.

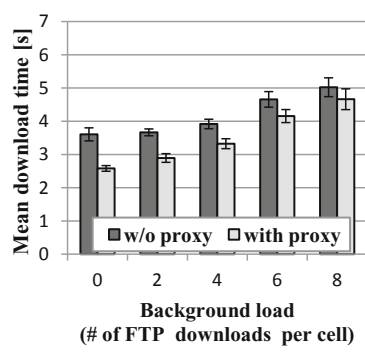

(a) $50 \mathrm{~ms}$

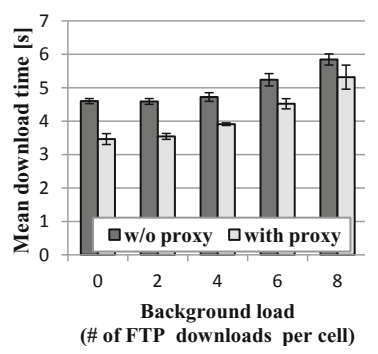

(b) $75 \mathrm{~ms}$

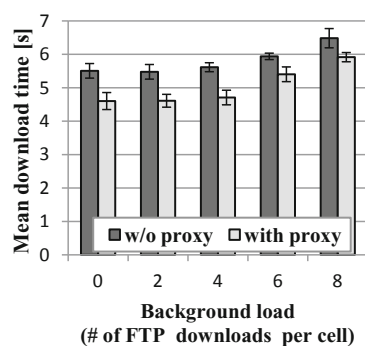

(c) $125 \mathrm{~ms}$

Fig. 7. Mean download time of web pages with and without a TCP proxy when the mean Internet delay is (a) $50 \mathrm{~ms}$, (b) $75 \mathrm{~ms}$ and (c) $125 \mathrm{~ms}$ 


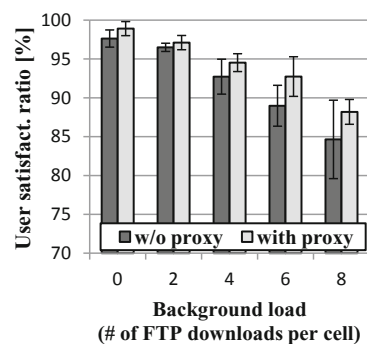

(a) $50 \mathrm{~ms}$

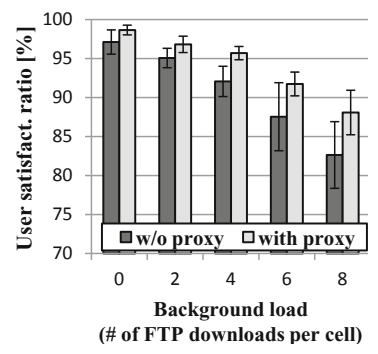

(b) $75 \mathrm{~ms}$

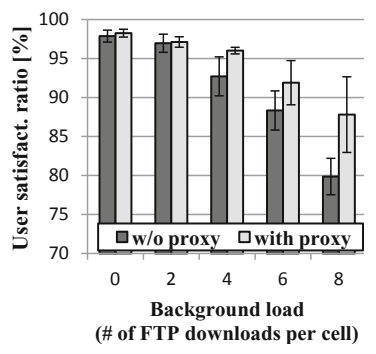

(c) $125 \mathrm{~ms}$

Fig. 8. The ratio of satisfactory and all video playbacks with and without a TCP proxy when the mean Internet delay is (a) $50 \mathrm{~ms}$, (b) $75 \mathrm{~ms}$ and (c) $125 \mathrm{~ms}$

Figure 7 (b) and (c) shows the same diagrams for higher Internet delays. As expected, the average download time increases with the mean Internet delay. However, TCP proxy can decrease the download time by the same amount (which is obviously a smaller ratio compared to the value of the download time), therefore the performance gain of TCP proxy decreases in case of web browsing as well when the Internet delay increases.

The performance of video streaming with and without TCP proxy is presented on Figure 8. The investigated conditions were the same as for the HTTP simulations. Each simulation case contained 228 video downloads and they were executed with 4 different random seeds, so from altogether 896 video downloads a user satisfaction ratio was calculated. The figures indicate that TCP proxy improves the user satisfaction ratio of video streaming in all the investigated conditions. The improvement is more profound when the Internet delay or the congestion level of the network is higher.

\section{Conclusion}

In this paper, it has been presented how the split connection TCP proxy concept can be used in LTE networks. Furthermore, it has been evaluated how the SAE-GW based TCP proxy affects the performance of bulk data transfer, web browsing and video download. Numerical results show that the TCP proxy can considerably increase the performance of web browsing and video download in all investigated conditions. TCP proxy is also able to significantly increase the performance of bulk data transfer if the transport network is not congested; however, there is no improvement when the transport is already fully utilized and the TCP proxy can even decrease the fairness in this case.

A possible extension of the work presented in this paper could be the evaluation of the eNB based TCP proxy solution. The study of the combined solution (two TCP proxies, one in the eNB and another in the SAE-GW) could also be an interesting follow-up topic. An additional research topic could be the fine tuning 
of the TCP parameters of the TCP proxy (e.g. initial window size) in a static or an adaptive way based on current network conditions.

\section{References}

1. 3GPP TS 25.814: Physical layer aspect for evolved Universal Terrestrial Radio Access, UTRA (2006)

2. Alexa: The top 500 sites on the web, http://www.alexa.com/topsites/

3. Baccelli, F., Carofiglio, G., Foss, S.: Proxy caching in split tcp: Dynamics, stability and tail asymptotics. In: The 27th Conference on Computer Communications, INFOCOM 2008, pp. 131-135. IEEE (April 2008)

4. Blaze: HTTP Pipelining - Big in Mobile, http://www.blaze.io/mobile/http-pipelining-big-in-mobile/

5. Border, J., Kojo, M., Griner, J., Montenegro, G., Shelby, Z.: RFC 3135: Performance Enhancing Proxies Intended to Mitigate Link-Related Degradations. Internet Engineering Task Force (June 2001)

6. Braden, R.: RFC 1122: Requirements for Internet Hosts - Communication Layers. Internet Engineering Task Force (October 1989)

7. Chakravorty, R., Katti, S., Pratt, I., Crowcroft, J.: Using tcp flow-aggregation to enhance data experience of cellular wireless users. IEEE Journal on Selected Areas in Communications 23(6), 1190-1204 (2005)

8. Dubois, E., Fasson, J., Donny, C., Chaput, E.: Enhancing tcp based communications in mobile satellite scenarios: Tcp peps issues and solutions. In: 2010 5th Advanced Satellite Multimedia Systems Conference (ASMA) and the 11th Signal Processing for Space Communications Workshop (SPSC), pp. 476-483 (September 2010)

9. Floyd, S., Henderson, T.: RFC 2582: The NewReno Modification to TCP's Fast Recovery Algorithm. Internet Engineering Task Force (April 1999)

10. Floyd, S., Jacobson, V.: Random early detection gateways for congestion avoidance. IEEE/ACM Trans. Netw. 1(4), 397-413 (1993)

11. Ha, S., Rhee, I., Xu, L.: CUBIC: a new TCP-friendly high-speed TCP variant. SIGOPS Oper. Syst. Rev. 42(5), 64-74 (2008)

12. Harri Holma, A.T.: LTE for UMTS: OFDMA and SC-FDMA Based Radio Access. Wiley (2009)

13. Hoque, M., Siekkinen, M., Nurminen, J.: On the energy efficiency of proxy-based traffic shaping for mobile audio streaming. In: 2011 IEEE Consumer Communications and Networking Conference (CCNC), pp. 891-895 (January 2011)

14. Ivanovich, M., Bickerdike, P., Li, J.: On tcp performance enhancing proxies in a wireless environment. IEEE Communications Magazine 46(9), 76-83 (2008)

15. Kwon, G.I., Byers, J.: Roma: reliable overlay multicast with loosely coupled tcp connections. In: Twenty-third AnnualJoint Conference of the IEEE Computer and Communications Societies. INFOCOM 2004, vol. 1, vol. (xxxv+2866), p. 4 (March 2004)

16. Ludwig, R., Katz, R.H.: The Eifel algorithm: making TCP robust against spurious retransmissions. SIGCOMM Comput. Commun. Rev. 30(1), 30-36 (2000)

17. Mascolo, S., Casetti, C., Gerla, M., Sanadidi, M.Y., Wang, R.: TCP westwood: Bandwidth estimation for enhanced transport over wireless links. In: MOBICOM, pp. 287-297 (2001) 
18. Matsumoto, M., Yamamoto, M.: Performance evaluation of flow fairness in proxybased tcp for ad hoc networks. In: 2011 IEEE Pacific Rim Conference on Communications, Computers and Signal Processing (PacRim), pp. 780-785 (August 2011)

19. Meyer, M., Sachs, J., Holzke, M.: Performance evaluation of a tcp proxy in wcdma networks. IEEE Wireless Communications 10(5), 70-79 (2003)

20. Moller, N., Molero, I., Johansson, K., Petersson, J., Skog, R., Arvidsson, A.: Using radio network feedback to improve tcp performance over cellular networks. In: 44th IEEE Conference on Decision and Control, 2005 and 2005 European Control Conference, CDC-ECC 2005, pp. 7434-7439 (December 2005)

21. Necker, M.C., Scharf, M., Weber, A.: Performance of Different Proxy Concepts in UMTS Networks. In: Kotsis, G., Spaniol, O. (eds.) Mobile and Wireless System. LNCS, vol. 3427, pp. 36-51. Springer, Heidelberg (2005), http://dx.doi.org/10.1007/978-3-540-31963-4_4

22. $\mathrm{Pu}, \mathrm{W}$. , Zou, Z., Chen, C.W.: New tcp video streaming proxy design for lasthop wireless networks. In: 2011 18th IEEE International Conference on Image Processing (ICIP), pp. 2225-2228 (September 2011)

23. Jain, R., Chiu, D., Hawe, W.: A Quantitative Measure Of Fairness And Discrimination For Resource Allocation In Shared Computer Systems. DEC Research Report TR-301 (September 1984)

24. Rao, A., Legout, A., Lim, Y.S., Towsley, D., Barakat, C., Dabbous, W.: Network characteristics of video streaming traffic. In: Proceedings of the Seventh Conference on Emerging Networking EXperiments and Technologies, CoNEXT 2011, pp. 25:125:12. ACM, New York (2011), http://doi.acm.org/10.1145/2079296.2079321

25. Ren, F., Huang, X., Liu, F., Lin, C.: Improving tcp throughput over hsdpa networks. IEEE Transactions on Wireless Communications 7(6), 1993-1998 (2008)

26. Ren, F., Lin, C.: Modeling and improving tcp performance over cellular link with variable bandwidth. IEEE Transactions on Mobile Computing 10(8), 1057-1070 (2011)

27. Shikama, T.: Mitigation of bursty packets by a tcp proxy improving tcp performance in a wired and wireless network. In: 2010 IEEE GLOBECOM Workshops (GC Wkshps), pp. 425-429 (December 2010)

28. Xylomenos, G., Polyzos, C., Mahonen, P., Saaranen, M.: TCP performance issues over wireless links. IEEE Communications Magazine 39(4), 52-58 (2001) 